\title{
Uso do Módulo Python Uncertainties no cálculo de incertezas experimentais da diferença de potencial e corrente elétrica de um protótipo experimental
}

Use of the Python Uncertainties Module in the calculation of experimental uncertainties of the potential difference and electric current of an experimental prototype

\author{
Flávio M. do Couto*国, Julio F. da Silva ${ }^{1}$ \\ ${ }^{1}$ Universidade Federal do Espírito Santo, Centro de Ciências Exatas, Naturais e da Saúde, Departamento de Química \\ e Física, Campus de Alegre, Alegre, ES, Brasil.
}

\begin{abstract}
Recebido em 29 de setembro de 2020. Revisado em 18 de janeiro de 2021. Aceito em 01 de fevereiro de 2021.
Nesse artigo, utilizou-se o Módulo Python Uncertainties no desenvolvimento dos códigos para efetuar os cálculos de incertezas experimentais, tanto para a diferença de potencial, quanto para as correntes elétricas. Para esse fim, foi desenvolvido um protótipo experimental que demonstra o efeito fotovoltaico a partir da utilização de materiais de baixo custo e, por conseguinte, com o objetivo de observar e quantificar as referidas grandezas físicas envolvidas. Além disso, foi realizada uma análise qualitativa e quantitativa do efeito fotovoltaico, assim como da Primeira Lei de Ohm. Nessa perspectiva, o protótipo foi associado a um multímetro, que permitiu a aferição direta da diferença de potencial, cujo o valor principal foi de $(0,4582 \pm 0,0004) \mathrm{V}$, quando incidido um feixe laser em um fotodiodo presente em um transistor $2 \mathrm{~N} 3055$, mas também os valores das correntes elétricas, sendo os valores principais de $(81,7100 \pm 0,1118) \mu \mathrm{A}$ e $(1,9990 \pm 0,0320) \mu \mathrm{A}$, quando se utilizou dos resistores ôhmicos de $1,0 \mathrm{k} \Omega$ e $220,0 \mathrm{k} \Omega$, respectivamente. Por fim, é importante salientar que essa proposta de artigo objetiva a anexação da computação física no cotidiano escolar, assim como promover o despertar científico na confecção de instrumentações específicas para o ensino-aprendizagem de física.
\end{abstract}

Palavras-chave: Python, Ensino de Física, Instrumentação para o Ensino de Física, Primeira Lei de Ohm.

In this article, the Python Uncertainties Module was used in the development of the codes to perform the calculations of experimental uncertainties, both for the potential difference and for the electric currents. For this purpose, an experimental prototype was developed that demonstrates the photovoltaic effect from the use of low-cost materials and, therefore, with the objective of observing and quantifying the referred physical quantities involved. In addition, a qualitative and quantitative analysis of the photovoltaic effect was carried out, as well as of Ohm's First Law. In this perspective, the prototype was associated with a multimeter, that allowed direct measurement of the potential difference, whose main value was $(0,4558 \pm 0,0004) \mathrm{V}$, when a laser beam was focused on a photodiode present in a 2N3055 transistor, but also the values of the electric currents, the main values being $(81,7100 \pm 0,1118) \mu \mathrm{A}$ and $(1,9990 \pm 0,0320) \mu \mathrm{A}$, when the ohmic resistors of $1,0 \mathrm{k} \Omega$ and 220,0 $\mathrm{k} \Omega$ were used, respectively. Finally, it is important to note that this article proposal aims at the annexation of physical computing in school routine, as well as promoting scientific awakening in the manufacture of specific instruments for teaching and learning physics.

Keywords: Python, Physics Teaching, Instrumentation for Physics Teaching, Ohm's First Law.

\section{Introdução}

A linguagem de programação em Python foi criada em 1990 por Guido Van Rossum. Destaca-se por ser open source (código aberto), além de apresentar elevado nível de desempenho e possuir uma gama de módulos préestabelecidos que permitem ao usuário desenvolver e alterar códigos para diversas finalidades [1]. O módulo Python Uncertainties pode ser utilizado na elaboração de expressões para cálculos simples e complexos de incertezas experimentais, via o método matemático de

\footnotetext{
*Endereço de correspondência: fmcfisico@gmail.com
}

derivadas [2]. Nesse artigo utilizou-se do módulo Python Uncertainties para o desenvolvimento de 4 (quatro) códigos específicos, a fim de utilizá-los no cálculo das incertezas experimentais da tensão e corrente elétrica, por meio de aplicação direta da Primeira Lei de Ohm, com uma precisão de saída configurada no código para um resultado de quatro (4) casas decimais definidas via método de arredondamento, em conformidade com os resultados apresentados nesse artigo. Nessa vertente, os valores obtidos, com a utilização de alguma instrumentação específica, seja ela analógica ou digital, são próximos de um resultado denominado valor principal e o intervalo de variação dos referidos valores é inversamente 
proporcional ao grau de confiabilidade atribuída àqueles mensurados devido as incertezas experimentais associadas a cada uma das medições [3, 4].

Partindo desse pressuposto, a equação geral da propagação de incertezas, permite reduzir a uma única faixa de valores todas as incertezas associadas ao processo de medição, o qual está atrelado as variáveis estimadas. A equação (1) sintetiza o formalismo mais geral no cálculo de propagação de incertezas, todavia se as variáveis primárias atreladas à grandeza física forem independentes, estiverem em consonância estatística e apresentarem os desvios individuais e padrão com a mesma ordem de grandeza para a mesma variável [4]. Além disso, é fundamental destacar que o método utilizado para o cálculo das incertezas experimentais via módulo Python Uncertainties está em ressonância com a referida equação 1 [3, 4]:

$$
\sigma_{\omega}^{2}=\left(\frac{\partial \omega}{\partial x}\right)^{2} \sigma_{x}^{2}+\left(\frac{\partial \omega}{\partial y}\right)^{2} \sigma_{y}^{2}+\cdots
$$

Nessa tocante, foi realizada a montagem de um protótipo experimental, cujos componentes eletrônicos estão listados na Tabela 1, a fim de aplicar o módulo Python Uncertainties, respectivamente, no cálculo das incertezas da Tensão, em conformidade com os dados descritos na Tabela 2 dois (2) deles no cálculo das correntes elétricas, cujos dados foram apresentados nas Tabelas 3 e 4 por fim, um código específico para análise e empregabilidade da Primeira Lei de Ohm no referido procedimento experimental. Os códigos e as descrições pormenorizadas dos mesmos, foram disponibilizados na íntegra via plataforma virtual GitHu ${ }^{1}$ [5].

Os objetivos elencados nesse artigo estão em ressonância com o desenvolvimento de uma instrumentação específica para a análise qualitativa e quantitativa do efeito fotovoltaico em um transistor; o desenvolvimento de códigos para o cálculo das incertezas das grandezas físicas supracitadas; aferição da corrente elétrica via Lei de Ohm. Por essas razões, vislumbrou-se a oportunidade e a necessidade de se desenvolver um protótipo experimental que pudesse ser acessível monetariamente, replicável e que oportunizasse abordar os principais conceitos relacionados à Primeira Lei de Ohm e ao estudo do efeito fotovoltaico dentro de um fotodiodo de silício (transistor) [6, 7]. Portanto, a proposição desse tipo de instrumentação no ensino de física, consideradas de baixo custo, tem merecido destaque em publicações científicas [8, 9. Nesse sentido, o protótipo desenvolvido tem a funcionalidade didática e específica para análises qualitativas e quantitativas acerca do efeito fotovoltaico, em que se destaca pelo ineditismo aplicado ao ensino de física, assim como o desenvolvimento dos códigos para

\footnotetext{
$\overline{1}$ O GitHub é um repositório de código aberto público desenvolvido para fins de compartilhamento, alocação de códigos, gerenciamento de projetos, dados e criação de softwares, por meio dos mais de 50 (cinquenta) milhões de desenvolvedores [5].
}

os cálculos das incertezas experimentais correlacionadas à ddp e corrente elétrica.

Uma vez realizada a montagem, calibragem e testes com o protótipo experimental, posteriormente, um conjunto de medidas foram realizadas para obtenção dos valores da diferença de potencial de forma direta com o auxílio de um multímetro, e com o mesmo, estimou-se a queda de corrente que passa por um resistor, a fim de obter valores da tensão elétrica de forma indireta via Primeira Lei de Ohm conforme a equação (2), onde $V$ diferença de potencial ou ddp $(\mathrm{V}) ; R$ - resistência ôhmica $(\Omega)$ e $i$ - corrente elétrica (A). [7]:

$$
V=R i
$$

Por conseguinte, foi realizado um estudo pormenorizado sobre a empregabilidade e desenvolvimento do módulo Python Uncertainties para o cálculo das incertezas experimentais para a Tensão (ddp) e as correntes elétricas, cujos códigos foram disponibilizados nos apêndices 1 a 4 e no GitHub ${ }^{1}$, nessa ordem, descritos previamente no resumo e, discutidos de maneira profícua na seção 3 desse artigo [2]. Portanto, com essa proposta espera-se elucidar atividades mais laborativas, a fim de estimular a criatividade, a participação efetiva do aluno na construção do seu conhecimento, nas tomadas de decisões, fundamentando-se em abordagens mais estruturadas, dinâmicas, alinhadas a promoção e o desenvolvimento de uma instrumentação adequada no estudo do efeito fotovoltaico, além de incentivar o uso da programação física no processo de ensino-aprendizagem [1, 10, 11]. Todavia, há uma gama de artigos que utilizam da fundamentação teórica do efeito fotovoltaico à aferição de grandezas físicas distintas, contudo aplicados na indústria, a citar: desenvolvimento de um veículo aéreo, não tripulado movido por energia solar para vigilância civil [12]; na determinação da eficiência energética em sistemas de iluminação [13]; na criação de algoritmos para a análise de sistemas fotovoltaicos [14.

Sendo assim, a utilização desse protótipo pode ser feita de modo didático em disciplinas distintas, porém correlacionadas, a citar uma de computação no ensino de física e outra que aborde os conteúdos acerca do efeito fotovoltaico. É fundamental destacar três relevantes aspectos, quanto à montagem e aplicação didática do referido protótipo. Primeiro, são componentes eletrônicos de fácil acesso e com baixo valor monetário comparados às instrumentações utilizadas nos laboratórios de instituições de ensino superior. Segundo, a programação utilizada nesse artigo é open source, ou seja, código aberto e gratuita, em que há várias plataformas digitais, sites, comunidades que a dominam e compartilham informações. Terceiro, exclusivamente para esse modelo apresentado (protótipo experimental), o código já foi desenvolvido e poderá ser replicado, uma vez que sua disponibilização será gratuita e livre. Portanto, reitera-se que a combinação e uso dessa proposta de instrumentação e linguagem de programação 
supracitadas poderão ser desenvolvidas em disciplinas de física e/ou engenharias, com enfoque no processo de ensino-aprendizagem de maneira didática.

\section{Protótipo Experimental e Metodologia de Aquisição de Dados}

A montagem do protótipo foi desenvolvida utilizando-se uma base suporte de polipropileno; uma chave on/off para o laser; um transistor 2N3055; ponteiras para fechar o circuito e efetuar a medição da tensão e corrente; fios na confecção do circuito elétrico; um resistor de $1,0 \mathrm{k} \Omega$ e outro de $220,0 \mathrm{k} \Omega$ disposto na parte inferior da base suporte; um laser de comprimento de onda estimado em $650 \mathrm{~nm}$, de acordo com as especificações do fabricante e um multímetro para aferição da diferença de potencial (tensão e/ou ddp) e corrente elétrica. A Figura 1 ilustra o referido protótipo utilizado para fins de estudo do efeito fotovoltaico.

Os resistores dispostos na parte inferior do suporte base, fazem parte de um circuito elétrico utilizado na obtenção da ddp, mas também da corrente elétrica. Isso é possível, por meio das ponteiras do multímetro, uma vez mescladas entre os terminais adequados, torna o circuito aberto ou fechado, a fim de mensurar as grandezas físicas de interesse. O circuito base proposto para a coleta de dados está ilustrado na Figura 2.

A instrumentação utilizada na confecção do referido protótipo, configura a ideia central de utilizar materiais de valor aquisitivo bem menor que os kits experimentais desenvolvidos por empresas especializadas, seja no Brasil

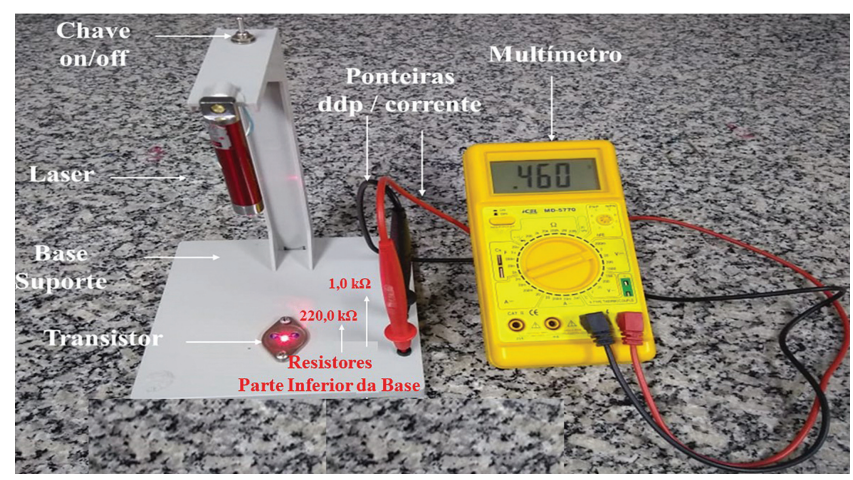

Figura 1: Protótipo para estudo do efeito fotovoltaico e a Primeira Lei de Ohm.

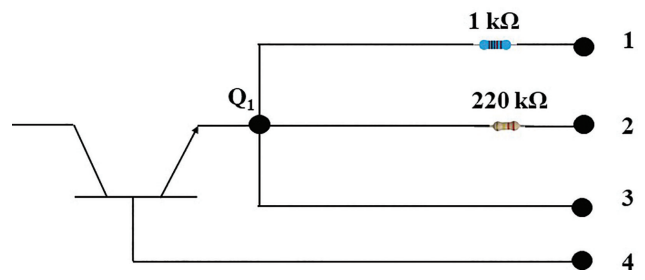

Figura 2: Circuito Elétrico utilizado na aferição da ddp e corrente elétrica.
Tabela 1: Componentes eletrônicos e a instrumentação utilizada na montagem do protótipo experimental. Valores estimados no mês de setembro do ano de 2020 .

\begin{tabular}{lc}
\hline $\begin{array}{l}\text { Componentes Eletrônicos e/ } \\
\text { ou Instrumentação }\end{array}$ & Valor Médio \\
\hline Multímetro ICEL MD-5770 & $\mathrm{R} \$ 90,00$ \\
Multímetro Instruterm MD-300 & $\mathrm{R} \$ 30,00$ \\
Transistor modelo 2N3055 Malaysia da & $\mathrm{R} \$ 5,00$ \\
marca ST & $\mathrm{R} \$ 5,00$ \\
Laser & $\mathrm{R} \$ 1,00$ \\
Resistores (1,0 a 220,0) $\mathrm{k} \Omega$ & $\mathrm{R} \$ 1,00$ \\
Terminais de Ponteiras (extremidades & \\
utilizadas na aferição dos dados) & \\
\hline
\end{tabular}

e/ou exterior. Tendo em vista esse aspecto, foram organizados na Tabela 1, os principais componentes eletrônicos e a instrumentação utilizada na montagem do protótipo experimental, consonantes aos seus valores médios no mercado.

Objetivou-se fazer a tomada de dados e comparar os resultados obtidos para os dois multímetros, uma vez comprovada a eficiência do multímetro de menor valor, este foi utilizado para fins da obtenção dos valores para a ddp e corrente elétrica descritos na seção resultados e discussões. Dessa forma, assegura-se um protótipo com valor menor ao usuário final, mantendose a qualidade e eficiência na aquisição dos dados. Em relação ao transistor, seu valor irrisório permite o estudo do efeito fotovoltaico, recorrendo ao foto diodo de silício localizado em seu interior, cuja exposição à incidência de luz, onde utilizou-se de um laser, cujo comprimento de onda e potência são, respectivamente, iguais $650 \mathrm{~nm} \mathrm{e}$ $5 \mathrm{~mW}$. Além disso, foram utilizados 2 (dois) resistores de $1,0 \mathrm{k} \Omega$ e $220,0 \mathrm{k} \Omega$, terminais de ponteiras (extremidades utilizadas na aferição dos dados), neste artigo utilizou-se 4 (quatro) terminais. Os valores estimados nesse artigo são referentes ao mês de setembro de 2020, todavia, estarão sujeitas as oscilações de preço do mercado.

O sistema foi alinhado, de maneira que, o feixe laser incidisse perpendicularmente sobre a fotocélula do transistor e, por conseguinte, foram mensuradas, de forma direta, a diferença de potencial nas extremidades 3 e 4 do circuito elétrico ilustrado na Figura 2 com o auxílio do multímetro na escala de $2000 \mathrm{~m}$ na função $D C V$. Sucessivamente, alternou-se a escala do multímetro para $200 \mu D C A$ e, consequentemente, foram obtidos os valores para a corrente elétrica nas extremidades 1 e 4 , onde foi possível fechar o circuito com um resistor de $1,0 \mathrm{k} \Omega$. A mesma escala foi utilizada nas extremidades 2 e 4 fechando o circuito com um resistor de $220,0 \mathrm{k} \Omega$.

\section{Resultados e Discussões}

Os procedimentos para aquisição dos dados pertinentes a ddp, consistiu na utilização das extremidades 3 e 4 do circuito elétrico, quando o feixe laser incidia sobre a 
fotocélula. Nesse sentido os valores para a ddp apareciam diretamente no visor do multímetro. Com isso, foram realizadas 100 medições, subdivididas em 20 (vinte) etapas, cada uma delas com 5 (cinco) repetições para o cálculo da ddp, devido ao efeito fotovoltaico. O valor médio com sua incerteza experimental ficou estimado em torno de $(\mathbf{0 , 4 5 8 2} \pm \mathbf{0 , 0 0 0 4}) \mathbf{V}$. O valor principal, assim como a sua incerteza experimental, foi calculado via módulo Python Uncertainties, cujo código completo pode ser apreciado gratuitamente na plataforma GitHUb [15]. Não obstante, os códigos foram desenvolvidos para obter uma precisão de quatro (4) casas decimais nos cálculos das incertezas e disponibilizados nos Apêndices de 1 a 4. Os resultados estão disponíveis na Tabela 2 e o código referente aos cálculos das incertezas está descrito, na íntegra, no Apêndice 1.

Os resultados dispostos na Tabela 3 são referentes a corrente elétrica, quando se utilizou o resistor ôhmico de $1,0 \mathrm{k} \Omega$. Para isso, foram realizadas 100 (cem) medições, subdividas em 20 (vinte) etapas, cada uma delas prevalecendo o total de 5 (cinco) repetições. Com isso, foi possível mensurar o valor principal e sua incerteza experimental de $(\mathbf{8 1 , 7 1 0} \pm \mathbf{0 , 1 1 2}) \mu \mathbf{A}$, ou seja, com uma variação de $\pm 0,14 \%$. Analogamente ao cálculo da ddp (Tabela 2), o código utilizado para obtenção dos valores presentes na Tabela 3 pode ser apreciado, na plataforma GitHUb [16]. O código referente aos cálculos das incertezas da Tabela 3 está descrito, na íntegra, no Apêndice 2.

Os resultados descritos na Tabela 4, são condizentes a utilização do resistor ôhmico de $220,0 \mathrm{k} \Omega$ e os procedimentos adotados, assim como a obtenção de todos os dados seguiram, de maneira fidedigna ao descrito na aquisição dos dados da Tabela 3. Contudo, obtevese o valor principal e sua incerteza experimental de $\mathbf{( 1 , 9 9 9 0} \pm \mathbf{0 , 0 3 2 0}) \boldsymbol{\mu A}$ com uma variação de $\pm 1,6 \%$. O código utilizado para obtenção dos valores presentes na Tabela 3 está na plataforma GitHUb [17]. O código referente aos cálculos das incertezas da Tabela 4 está descrito, na íntegra, no Apêndice 3.

Em seguida, foram substituídos os valores da ohmicidade de 1,0 k $\Omega$ e da corrente elétrica na Equação (2). Essas substituições permitiram obter o valor principal para a diferença de potencial e sua incerteza experimental $(0,0830 \pm 0,0042) \mathrm{V}[18$. Comparando este valor com aquele demonstrado na Tabela 2 observou-se uma discrepância considerável. Sendo assim, justifica-se esse fato, por consequência do baixo valor da corrente elétrica que percorre o circuito elétrico descrito na Figura 2 Contudo, a utilização do referido resistor se torna insignificante. Isso permitiu estabelecer os valores na função do multímetro em DCA e escala de $200 \mu \mathrm{A}$ ao inserir as ponteiras de prova nos terminais 3 e 4 do circuito, a fim de obter os mesmos valores de corrente, previamente, mensurados via os terminais 1 e 4 , ratificando dessa forma, a inutilização do resistor. De maneira semelhante, foi substituído os valores da resistência de $220,0 \mathrm{k} \Omega$ e da corrente elétrica na Equação (2). Isso permitiu obter de forma indireta o valor principal da diferença de potencial, associado à sua incerteza experimental de $(0,4438 \pm \mathbf{0 , 0 2 3 3 )} \mathrm{V}[18$.

A incerteza experimental detém uma gama de valores dispersos em torno de um valor de referência, ou seja, denominado valor principal. Observa-se essa variação

Tabela 2: Valores da ddp para o Efeito Fotovoltaico.

\begin{tabular}{|c|c|c|c|c|c|c|c|}
\hline Medida & $\mathrm{T} 1(\mathrm{~V})$ & $\mathrm{T} 2(\mathrm{~V})$ & T3(V) & $\mathrm{T} 4(\mathrm{~V})$ & T5 (V) & $\begin{array}{l}\text { Médias das } \\
\text { ddp's (V) }\end{array}$ & $\begin{array}{c}\text { Incerteza } \\
(\mathrm{V})\end{array}$ \\
\hline 1 & 0,4630 & 0,4620 & 0,4590 & 0,4580 & 0,4620 & 0,4608 & \\
\hline 2 & 0,4600 & 0,4630 & 0,4640 & 0,4580 & 0,4600 & 0,4610 & \\
\hline 3 & 0,4620 & 0,4590 & 0,4590 & 0,4580 & 0,4590 & 0,4594 & \\
\hline 4 & 0,4630 & 0,4610 & 0,4610 & 0,4610 & 0,4610 & 0,4614 & \\
\hline 5 & 0,4620 & 0,4570 & 0,4600 & 0,4590 & 0,4600 & 0,4596 & \\
\hline 6 & 0,4580 & 0,4540 & 0,4540 & 0,4540 & 0,4540 & 0,4548 & \\
\hline 7 & 0,4550 & 0,4570 & 0,4560 & 0,4560 & 0,4570 & 0,4562 & \\
\hline 8 & 0,4570 & 0,4570 & 0,4560 & 0,4560 & 0,4560 & 0,4564 & \\
\hline 9 & 0,4570 & 0,4570 & 0,4570 & 0,4570 & 0,4570 & 0,4570 & \\
\hline 10 & 0,4560 & 0,4560 & 0,4560 & 0,4560 & 0,4560 & 0,4560 & $\pm 0,0024$ \\
\hline 11 & 0,4570 & 0,4560 & 0,4590 & 0,4580 & 0,4570 & 0,4574 & \\
\hline 12 & 0,4580 & 0,4590 & 0,4590 & 0,4590 & 0,4590 & 0,4588 & \\
\hline 13 & 0,4570 & 0,4580 & 0,4570 & 0,4570 & 0,4580 & 0,4574 & \\
\hline 14 & 0,4600 & 0,4590 & 0,4580 & 0,4580 & 0,4580 & 0,4586 & \\
\hline 15 & 0,4580 & 0,4580 & 0,4560 & 0,4580 & 0,4580 & 0,4576 & \\
\hline 16 & 0,4580 & 0,4590 & 0,4580 & 0,4580 & 0,4580 & 0,4582 & \\
\hline 17 & 0,4580 & 0,4590 & 0,4590 & 0,4580 & 0,4590 & 0,4586 & \\
\hline 18 & 0,4580 & 0,4590 & 0,4580 & 0,4580 & 0,4580 & 0,4582 & \\
\hline 19 & 0,4600 & 0,4590 & 0,4590 & 0,4570 & 0,4590 & 0,4588 & \\
\hline 20 & 0,4590 & 0,4580 & 0,4580 & 0,4580 & 0,4580 & 0,4582 & \\
\hline \multicolumn{8}{|l|}{ Valor } \\
\hline $\begin{array}{l}\text { Principal } \\
\text { da ddp }\end{array}$ & - & - & - & - & - & $0,4582 \pm$ & 0,0005 \\
\hline
\end{tabular}


Tabela 3: Valores da corrente elétrica para o resistor ôhmico de $1,0 \mathrm{k} \Omega$.

\begin{tabular}{lcccccc}
\hline Medida & $\mathbf{C 1}(\boldsymbol{\mu A})$ & $\mathbf{C 2}(\boldsymbol{\mu A})$ & $\mathbf{C 3}(\boldsymbol{\mu A})$ & $\mathbf{C 4}(\boldsymbol{\mu A})$ & $\mathbf{C 5}(\boldsymbol{\mu A})$ & $\begin{array}{c}\text { Média das } \\
\text { Corrétricas }(\boldsymbol{\mu A})\end{array}$ \\
\hline $\mathbf{1}$ & 94,3000 & 90,4000 & 89,1000 & 88,0000 & 86,5000 & $89,6600 \pm 0,5353$ \\
$\mathbf{2}$ & 83,3000 & 82,4000 & 81,9000 & 81,9000 & 80,5000 & $82,0000 \pm 0,5009$ \\
$\mathbf{3}$ & 85,5000 & 83,2000 & 81,8000 & 81,4000 & 80,7000 & $82,5200 \pm 0,5033$ \\
$\mathbf{4}$ & 87,0000 & 83,3000 & 81,2000 & 80,0000 & 77,6000 & $81,8200 \pm 0,5003$ \\
$\mathbf{5}$ & 80,2000 & 79,5000 & 79,1000 & 78,9000 & 78,5000 & $79,2400 \pm 0,4885$ \\
$\mathbf{6}$ & 82,2000 & 79,7000 & 78,2000 & 77,3000 & 77,2000 & $78,9200 \pm 0,4872$ \\
$\mathbf{7}$ & 82,4000 & 78,1000 & 74,1000 & 75,1000 & 74,3000 & $76,8000 \pm 0,4778$ \\
$\mathbf{8}$ & 80,3000 & 79,9000 & 77,0000 & 76,3000 & 76,1000 & $77,9200 \pm 0,4827$ \\
$\mathbf{9}$ & 81,9000 & 81,0000 & 79,9000 & 79,4000 & 78,8000 & $80,2000 \pm 0,4929$ \\
$\mathbf{1 0}$ & 80,9000 & 80,0000 & 79,2000 & 78,7000 & 78,1000 & $79,3800 \pm 0,4892$ \\
$\mathbf{1 1}$ & 91,7000 & 87,7000 & 90,1000 & 89,6000 & 88,5000 & $89,5200 \pm 0,5345$ \\
$\mathbf{1 2}$ & 88,6000 & 86,7000 & 90,1000 & 89,3000 & 80,3000 & $87,0000 \pm 0,5235$ \\
$\mathbf{1 3}$ & 77,0000 & 76,4000 & 86,6000 & 81,6000 & 84,7000 & $81,2600 \pm 0,4979$ \\
$\mathbf{1 4}$ & 83,5000 & 85,1000 & 80,0000 & 77,9000 & 77,6000 & $80,8200 \pm 0,4958$ \\
$\mathbf{1 5}$ & 79,8000 & 79,0000 & 81,4000 & 81,2000 & 81,4000 & $80,5600 \pm 0,4945$ \\
$\mathbf{1 6}$ & 86,5000 & 88,1000 & 86,7000 & 86,7000 & 87,4000 & $87,0800 \pm 0,5236$ \\
$\mathbf{1 7}$ & 76,3000 & 76,3000 & 76,6000 & 77,8000 & 79,3000 & $77,2600 \pm 0,4797$ \\
$\mathbf{1 8}$ & 83,4000 & 82,6000 & 83,2000 & 82,7000 & 81,0000 & $82,5800 \pm 0,5035$ \\
$\mathbf{1 9}$ & 78,4000 & 78,7000 & 77,9000 & 78,6000 & 80,4000 & $78,8000 \pm 0,4866$ \\
$\mathbf{2 0}$ & 80,3000 & 81,5000 & 81,5000 & 80,7000 & 80,3000 & $80,8600 \pm 0,4958$ \\
\hline Valor & - & - & - & - & & $\mathbf{8 1 , 7 1 0 0} \pm \mathbf{0 , 1 1 1 8}$ \\
Principal & & & & & & \\
da Corrente & & & & & & \\
\hline
\end{tabular}

Tabela 4: Valores da corrente elétrica para o resistor ôhmico de 220,0 k $\Omega$.

\begin{tabular}{lcccccc}
\hline Medida & C1 $(\boldsymbol{\mu A})$ & $\mathbf{C 2}(\boldsymbol{\mu A})$ & $\mathbf{C 3}(\boldsymbol{\mu A})$ & $\mathbf{C 4}(\boldsymbol{\mu A})$ & $\mathbf{C 5}(\boldsymbol{\mu A})$ & Média $(\boldsymbol{\mu A})$ \\
\hline $\mathbf{1}$ & 2,0000 & 1,9000 & 2,0000 & 2,0000 & 2,0000 & $1,9800 \pm 0,1430$ \\
$\mathbf{2}$ & 2,0000 & 2,1000 & 2,0000 & 2,0000 & 1,9000 & $2,0000 \pm 0.1431$ \\
$\mathbf{3}$ & 2,1000 & 2,0000 & 1,9000 & 2,0000 & 2,0000 & $2,0000 \pm 0,1431$ \\
$\mathbf{4}$ & 2,0000 & 2,0000 & 2,0000 & 2,0000 & 2,0000 & $2,0000 \pm 0,1431$ \\
$\mathbf{5}$ & 2,1000 & 2,0000 & 2,0000 & 2,0000 & 2,0000 & $2,0200 \pm 0,1432$ \\
$\mathbf{6}$ & 1,9000 & 2,0000 & 2,1000 & 2,0000 & 2,0000 & $2,0000 \pm 0,1431$ \\
$\mathbf{7}$ & 2,0000 & 1,9000 & 1,9000 & 2,0000 & 1,9000 & $1,9400 \pm 0,1428$ \\
$\mathbf{8}$ & 2,0000 & 2,0000 & 2,0000 & 2,0000 & 2,0000 & $2,0000 \pm 0,1431$ \\
$\mathbf{9}$ & 2,0000 & 2,0000 & 2,0000 & 1,9000 & 2,1000 & $2,0000 \pm 0,1431$ \\
$\mathbf{1 0}$ & 2,1000 & 2,0000 & 2,0000 & 2,0000 & 2,0000 & $2,0200 \pm 0,1432$ \\
$\mathbf{1 1}$ & 2,0000 & 2,1000 & 2,1000 & 1,9000 & 2,0000 & $2,0200 \pm 0,1432$ \\
$\mathbf{1 2}$ & 2,0000 & 2,0000 & 2,0000 & 2,0000 & 2,0000 & $2,0000 \pm 0,1431$ \\
$\mathbf{1 3}$ & 2,1000 & 2,0000 & 2,0000 & 2,0000 & 2,0000 & $2,0200 \pm 0,1432$ \\
$\mathbf{1 4}$ & 2,0000 & 2,0000 & 2,0000 & 2,0000 & 2,0000 & $2,0000 \pm 0,1431$ \\
$\mathbf{1 5}$ & 2,0000 & 2,0000 & 2,0000 & 2,0000 & 2,0000 & $2,0000 \pm 0,1431$ \\
$\mathbf{1 6}$ & 2,0000 & 1,9000 & 2,1000 & 1,9000 & 2,1000 & $2,0000 \pm 0,1431$ \\
$\mathbf{1 7}$ & 1,9000 & 2,0000 & 2,0000 & 2,0000 & 2,0000 & $1,9800 \pm 0,1430$ \\
$\mathbf{1 8}$ & 2,1000 & 2,0000 & 2,1000 & 2,0000 & 2,0000 & $2,0400 \pm 0,1433$ \\
$\mathbf{1 9}$ & 2,0000 & 2,0000 & 2,0000 & 1,9000 & 2,0000 & $1,9800 \pm 0,1430$ \\
$\mathbf{2 0}$ & 2,0000 & 1,9000 & 2,0000 & 2,0000 & 2,0000 & $1,9800 \pm 0,1430$ \\
\hline Valor & - & - & - & - & - & $\mathbf{1 , 9 9 9 0} \pm \mathbf{0 , 0 3 2 0}$ \\
Principal & & & & & & \\
da Corrente & & & & & & \\
\hline
\end{tabular}

com cerca de $\pm 5,3 \%$ em relação ao valor principal. Não obstante, existem fatores intrínsecos no processo de aquisição de dados em o todo procedimento experimental, dos quais o resultado final depende diretamente da precisão, calibração e a incerteza das instrumentações utilizadas, assim como o alinhamento do sistema e o fator humano na execução das medições [3]. O modelo adotado nesse artigo foi para o cálculo das incertezas experimentais, tendo em vista que os valores da tensão obtidos e informados na Tabela 2, são utilizados como referência, pois são variáveis considerando as fontes de erros supracitadas, mesmo vinculados a um percentual diminuto. Sendo assim, a prerrogativa de não ter um referencial imutável e/ou fixo, faz com que se utilize da 
teoria de incertezas experimentais, em contrapartida à teoria de erros.

Notoriamente, esse valor $(\mathbf{0 , 4 4 3 8} \pm \mathbf{0 , 0 2 3 3}) \mathrm{V}$ é condizente àquele calculado na Tabela 2, o qual corrobora a faixa de valores associados a sua incerteza experimental. O código referente aos cálculos das incertezas está descrito, na íntegra, no Apêndice 4.

\section{Conclusões}

Em geral, as análises via código de programação por meio do módulo Python Uncertainties, podem proporcionar uma visão mais holística do uso da computação no ensino de física, por meio da linguagem em Python, dentre tantas outras open source, como um recurso gratuito e relevante no enriquecimento do processo de ensino-aprendizagem [19].

A instrumentação desenvolvida nesse artigo prevê a possibilidade de ser usada em modo didático com a utilização de poucos recursos financeiros e construir um protótipo eficaz no estudo da Primeira Lei de Ohm e na observação do efeito fotovoltaico de maneira qualitativa, mas também quantitativa. Nessa tocante, a obtenção de dados referentes a diferença de potencial e a corrente elétrica. Essas análises em consonância com os dados apresentados corroboram, os códigos desenvolvidos sinalizam uma notória eficiência e, posterior, aplicação e replicabilidade em instituições de ensino básica e/ou superior.

\section{Agradecimentos}

À Universidade Federal do Espírito Santo.

À Fundação de Amparo à Pesquisa e Inovação do Espírito Santo - FAPES.

\section{Material Suplementar}

O seguinte material suplementar está disponível online: Apêndice 1

Apêndice 2

Apêndice 3

Apêndice 4

\section{Referências}

[1] A. Downey, J. Elkner e C. Meyers, How to Think Like a Computer Scientist (Green Tea Press Needham, Massachusetts, 2002).

[2] E.O. Lebigot, Uncertainties Python Package: Documentation Release 3.0.1, disponível em: https://pythonhost ed.org/uncertainties, acessado em 19/08/2020.

[3] J.H. Vuolo, Fundamentos da teoria de erros (Edgard Blucher, São Paulo, 1996), $2^{a}$ ed.

[4] P. Lima Jr. e F.L. da Silveira, Rev. Bras. Ens. Fis. 33, 2 (2011).

[5] https://github.com/, acessado em 17/01/2021.
[6] M. Azevedo e A. Cunha, Fazer uma célula fotovoltaica, disponível em: https://www.academia.edu/25810777/ Physics_on_Stage_Fazer_uma_c\%C3\%A9lula_fotov oltaica

[7] M. Alonso e E.J. Finn, Física (Escolar Editora, Forte da Casa, 2012).

[8] A.J. Bühler, Estudo de técnicas de determinação experimental e pós processamento de curvas características de módulos fotovoltaicos. Tese de Doutorado, Universidade Federal do Rio Grande do Sul, Porto Alegre (2011).

[9] A.M.F. Diniz e R.D. Araújo, Revista Brasileira de Ensino de Física 41, e20180216 (2019).

[10] T. Igoe e D. O'sullivan, Physical Computing - Sensing and Controlling the Physical World with Computers (Thomson Course Technology, Boston, 2004).

[11] N.J. Giordano e H. Nakanishi, Computational Physics (Pearson, London, 2005), $2^{\mathrm{a}}$ ed.

[12] N.J.P. Betancourth, J.E.P. Villamarin, J.J.V. Rios, P.D. Bravo-Mosquera e H.D. Cerón-Muñoz, J. Aerosp. Technol. Manag. 8, 385 (2016).

[13] C.L. Boquimpani, A.L.T.S. Motta, O.L.C. Souza e C.H.V.R. Oliveira, Ambient. constr. 19, 303 (2019).

[14] G. Lopes Filho, R.A.P. Franco e F.H.T. Vieira, TEMA (São Carlos) 21, 157 (2020).

[15] https://github.com/julio-fs/Calculos-estatasticos-para - corrente-tensao-e-1--Lei-de-Ohm/blob/master/Estatis ticadaTensao.ipynb, acessado em 18/01/2021.

[16] https://github.com/julio-fs/Calculos-estatasticos-para - corrente-tensao-e-1--Lei-de-Ohm/blob/master/Estatis ticaCorrente1.ipynb, acessado em 18/01/2021.

[17] https://github.com/julio-fs/Calculos-estatasticos-para -corrente-tensao-e-1--Lei-de-Ohm/blob/master/Estatis ticaCorrente2.ipynb acessado em 18/01/2021.

[18] https://github.com/julio-fs/Calculos-Estatasticos-pa ra-Corrente-Tensao-e-Primeira-Lei-de-Ohm/blob/ma ster/CalculosPrimeiraLeideOhm.ipynb, acessado em $18 / 01 / 2021$

[19] L.E. Borges, Python para Desenvolvedores (Novatec Editora, Rio de Janeiro, 2010), $2^{\mathrm{a}}$ ed. 